Canadian

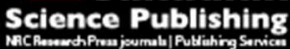

Applied Physiology, Nutrition, and Metabolism Physiologie appliquée, nutrition et métabolisme

\title{
Food sources of sodium, saturated fat and added sugar in the Physical Activity and Nutrition for Diabetes in Alberta (PANDA) Trial
}

\begin{tabular}{|r|l|}
\hline Journal: & Applied Physiology, Nutrition, and Metabolism \\
\hline Manuscript ID & apnm-2017-0266.R1 \\
\hline Manuscript Type: & Article \\
\hline Date Submitted by the Author: & 04-Jul-2017 \\
\hline $\begin{array}{r}\text { Complete List of Authors: } \\
\text { Nutritional Science } \\
\text { Chan, Catherine; University of Alberta, }\end{array}$ & $\begin{array}{l}\text { Asaad, Ghada; University of Alberta, Department of Agricultural, Food and } \\
\text { consideration in a Special } \\
\text { Issue? : }\end{array}$ \\
\hline Keyword: & Sodium, Saturated fat \\
\hline & \\
\hline
\end{tabular}




\section{Title page}

Food sources of sodium, saturated fat and added sugar in the Physical Activity and Nutrition for Diabetes in Alberta (PANDA) Trial

Ghada Asaad ${ }^{1 *}$ and Catherine B. Chan ${ }^{1,2,3}$

${ }^{1}$ Department of Agricultural, Food and Nutritional Science, University of Alberta, Edmonton, AB T6G 2R3, Canada

${ }^{2}$ Department of Physiology, University of Alberta, Edmonton, AB T6G 2R3, Canada

${ }^{3}$ Diabetes, Obesity and Nutrition Strategic Clinical Network, Alberta Health Services, Edmonton, AB T6G 2R3, Canada

* Corresponding author: gasaad@ualberta.ca 


\begin{abstract}
Diabetic patients may find it difficult to achieve recommended nutrient intakes embedded within dietary guidelines. The objective of this analysis was to document total sodium, saturated fat and added sugar intake as well as the main food sources of these nutrients in Canadian adults with type 2 diabetes before and after an intervention focused on healthy eating. Participants were enrolled in a single-arm dietary intervention trial designed to improve glycemic control and adherence to dietary recommendations. A 4-week menu plan and recipes were provided for participants along with a 6-week educational curriculum. Three repeated 24-hour dietary recalls were collected at baseline and 3 months. Food sources of sodium, saturated fat and added sugar were a secondary outcome derived from the dietary recalls. After 3 months, there was a reduction $(p<0.05)$ in sodium intake of $561 \mathrm{mg} /$ day, mainly due to reduced consumption of processed meats, soups and condiments. Significantly lower intake of processed meat contributed to -2.9 $\mathrm{g} /$ day saturated fat intake $(\mathrm{p}<0.1)$ while added sugar intake declined by $7 \mathrm{~g} /$ day $(\mathrm{p}<0.1)$, due to lower consumption of baked goods/desserts and chocolate (both $p<0.05$ ). The intervention was beneficial for type 2 diabetes patients in terms of changing dietary habits. However, the majority of the participants still exceeded the dietary guidelines for sodium and saturated fat. In addition to the efforts of individuals and their healthcare providers, strategies to increase the nutritional quality of prepared foods could provide widespread benefits.
\end{abstract}

Key words: Sodium, Saturated fat, Added sugars, Food sources, Diabetes intervention 


\section{Introduction}

Diabetes is a challenging disease to monitor and manage, requiring significant patient attention to self-care activities, including dietary modifications (Dworatzek et al, 2013). Non-adherence to dietary recommendations is an issue that has been extensively documented (Thanopoulou et al, 2004; Eilat-Adar et al, 2008; Rivellese et al., 2008; Muñoz-Pareja et al, 2012; Coyle et al., 2013; Asaad et al., 2016). Influences contributing to therapeutic non-compliance in several disease states have been reviewed and include factors related to the patient, type of therapy, socioeconomic status, the healthcare system, and disease severity (Jin et al., 2008). Nutrition therapy is crucial for successful diabetes management. Making changes in the dietary pattern of diabetic patients leads to reductions in glycated hemoglobin (A1C) of 1.0-2.0\%, improved serum cholesterol levels, and enhanced weight management (Dworatzek et al, 2013). On the other hand, poor glycemic control is associated with macrovascular and microvascular complications in diabetic patients such as heart disease, stroke, kidney disease, and nerve damage (Stratton et al., 2009).

Evidence links food and dietary patterns to the risk of developing type 2 diabetes (Ley et al., 2016). Sugar (Rippe and Angelopoulos 2016) and fat intake (Ericson et al., 2015), and an unhealthy/western dietary pattern characterized by high intakes of refined carbohydrate, processed meat, and fried food (McEvoy et al., 2014) are associated with increased risk of type 2 diabetes. Individual nutrients and diet patterns also play a role in diabetes management. Sucrose intake more than $10 \%$ of daily total energy may increase blood glucose and triglycerides in people with type 2 diabetes (Dworatzek et al, 2013). Saturated fats should be restricted to less than $7 \%$ of total daily energy due to the high 
risk of coronary artery disease in people with type 2 diabetes (Dworatzek et al, 2013). There is no specific recommendation for sodium intake according to the Canadian Diabetes Association (Dworatzek et al, 2013), but the Dietary Approaches to Stop Hypertension trial has shown that moderate daily sodium intake $(<2400 \mathrm{mg})$ decreased systolic and diastolic blood pressure, as well as decreasing A1C, and had beneficial effects on cardiometabolic risks (Azadbakht et al., 2011). The tolerable upper intake limit (UL) for adults in Canada is $2300 \mathrm{mg} /$ day (Health Canada, 2017).

Identifying common food sources of nutrients in the diet of the general population is valuable because it offers experts the ability to determine potential benefits to improve diet quality, promote health and prevent disease (The National Academy of Sciences, 1988). Health Canada collects longitudinal data on food and nutrient consumption for the Canadian population to provide information on health determinants, health status, and health system utilization (Health Canada, 2006), but publications related to the diabetic sub-population are limited and do not always include food sources (Shi et al., 2011; Langlois and Garrigue, 2012). Furthermore, the most recent data are from 2004 (Health Canada, 2006) and may not reflect current eating trends. A determination of food sources in a diabetic population could be beneficial to identify and focus intervention efforts on unhealthy diet patterns and foods in order to improve glycemic control and health outcomes. Certain approaches to improve dietary patterns may lend themselves to reducing processed foods, which are typically high in sodium, fat or sugar. For example, menu planning may assist people with diabetes to adhere to a healthy diet (SoriaContreras et al., 2012; Asaad et al., 2016) while provision of simple recipes may also be 
of benefit because studies of other populations have shown that home-cooking improves diet quality (Wolfson and Bleich, 2015).

Research in Spain identified the sources of food that contributed most to sodium, saturated fat and added sugar in people with chronic diseases including type 2 diabetes (Guallar-Castillón et al., 2013). Generalizability of the outcomes is limited by cultural specificity of dietary patterns, among other factors. To our knowledge, no previous studies have analyzed food sources of sodium, saturated fat, and added sugar or the impact of a dietary intervention on those foods in Canadian adults with diabetes. The Physical Activity and Nutrition for Diabetes in Alberta (PANDA) trial focused on healthy eating with provision of menu plans and recipes as primary education tools. We previously showed that 12-week implementation of the PANDA intervention reduced A1C by $0.7 \%$, and had beneficial effects on cholesterol and blood pressure. Participants had reduced intake of calories, added sugar and sodium but saturated fat consumption was stable (Asaad et al., 2016). The objectives of this secondary analysis were to identify primary food sources of sodium, saturated fat, and added sugar at baseline and how each was changed by participation in the PANDA intervention study after 3 months. We hypothesized that intakes of these nutrients from processed foods would be reduced after the intervention.

\section{Materials and methods}

\section{Study sample and intervention}

The PANDA study was a single-arm nutrition intervention (ClinicalTrials.gov registration NCT01625507). The primary outcomes were dietary adherence to recommendations and change in A1C. Details of the study design, participant recruitment 
and primary outcomes are reported elsewhere (Asaad et al., 2016). We included participants between 35-80 years of age, who had been diagnosed with type 2 diabetes (self-report), were able to read and communicate in English, and attend meetings at the study site. Potential participants were screened by phone by the study coordinator to exclude those with concomitant diseases or conditions that would preclude following the menu plan, type 1 diabetes, severe diabetes complications or pregnancy. Sample size was calculated to detect a clinically relevant, $0.5 \%$ change in hemoglobin A1c concentration (alpha $=0.05$, beta $=0.8$, paired t-test) and to account for a drop-out rate of $30 \%$ based on a pilot study (Soria-Contreras et al., 2014). At baseline, participants completed a series of demographic and diabetes care-related questionnaires, anthropometric measures, and had blood sampled for A1c and lipid analyses.

Details of the PANDA dietary intervention were presented elsewhere (Asaad et al., 2016). The 3-month intervention was delivered by a dietitian to 5 small groups (1214/group) of participants who received a 4-week menu plan with recipes and 6 experiential education sessions focused on healthy eating strategies. The first session was reviewed Eating Well with Canada's Food Guide and serving sizes. The second session introduced the menu plan and the third focused on adapting the menu plan for energy needs, family size and cultural preferences. The fourth session addressed food choices when dining out and reading food labels. The fifth session was about carbohydrate counting and low glycemic food. The last session was a grocery store tour with a dietitian. The menus were constructed to meet the recommendations of Eating Well with Canada's Food Guide and with saturated fat $\leq 7 \%$ of total energy. The amount of added 
sugar or sodium was not calculated at the time of menu plan development; however, the menus were designed to be relatively low in added sugar and salt.

\section{Dietary assessment}

Changes in dietary intake over the intervention period were measured by 3 repeated 24hour dietary recalls (collected on 2 weekdays and 1 weekend day) using WebSpan, an internet-based questionnaire, completed by participants at baseline and 3-months. WebSpan utilization has been shown to reduce assessment error and bias (Storey and McCargar, 2012). Dietary data were checked for accuracy by a trained graduate student. Duplicate entries of a single food item were removed. The WebSpan database utilizes the Canadian Nutrient File database (2001, Health Canada, Ottawa, ON, Canada) to generate sodium and saturated fat values of foods, corrected for portion size. The WebSpan software does not calculate added sugar. Added sugar (fructose plus sucrose) was calculated manually from a database developed for commonly consumed Canadian foods based on the ESHA database with additional foods added by evaluation of manufacturer's food labels (Durai Raj et al., 2017). To determine the contribution of food sources to sodium, saturated fat, and added sugar intake, all foods were classified into groups based on relatively homogenous composition (Table 1) adapted from Guallar-Castillón (Guallar-Castillón et al., 2013). The contribution that specific categories made to the percentage of total sodium, saturated fat and added sugar daily intake was calculated. Consumption from each category was represented as a percentage of total daily intake. Participants were excluded from the analysis if they did not complete the three 24-hour dietary recalls pre- and post-intervention or reported consumption of implausible total energy values (outside the range of 500-3500 kcal/d for women and 800-4000 kcal/d for 
men) (Willet, 1998). Among 73 participants enrolled in the PANDA intervention, $n=61$ were included in the analysis, and none were excluded for implausible energy intake (Willet, 1998).

\section{Statistical analysis}

The contribution that specific categories of food made to the daily percentage intake of total sodium, saturated fat, and added sugar was calculated as (nutrient content of the food classification (Table 1) summed for all individuals/total daily intake of target nutrient from all foods for all individuals)*100\% (Guallar-Castillón et al., 2013). Gender differences in total intake were compared by unpaired t-test. To evaluate the effectiveness of the intervention, a paired t-test was used to compare the change in food sources of sodium, saturated fat, and added sugar pre- and post-intervention. Chi-squared tests were used to explore differences in the number of participants who met nutrition recommendations pre- and post-intervention. Analyses were performed using IBM SPSS version 22 (SPSS Inc., Chicago, IL, USA), and statistical significance was determined at $\mathrm{p}<0.05$. Because the study was not initially powered to detect changes in food sources, trends in consumption were also noted if $\mathrm{p}<0.1$.

\section{Results}

The 61 participants included in food source analyses included 32 males and 29 females, with an average age of $59.2 \pm 9.5$ years, duration of diabetes of $9.3 \pm 8.6$ years and the majority were Caucasian (85.2\%). Baseline A1c was $7.9 \pm 1.7 \%$, BMI $32.4 \pm 7.0 \mathrm{~kg} / \mathrm{m}^{2}$, systolic blood pressure $128 \pm 14 \mathrm{mmHg}$, and diastolic blood pressure $78 \pm 9 \mathrm{mmHg}$. At baseline, average daily sodium and saturated fat intakes exceeded recommendations, whereas added sugar intake met the recommendation (Table 2). After the intervention 
there was a significant $(\mathrm{p}<0.01)$ reduction in daily sodium intake and sodium density with trends $(\mathrm{p}<0.1)$ to reduced saturated fat and added sugar intakes (Table 2).

Table 3 displays the primary food sources of sodium before and after the PANDA intervention. Bread was the major source of sodium for diabetic participants. Other important sources of sodium included processed foods (luncheon meats, soups, condiments) and grain based mixed foods such as pasta dishes. The total contribution of sodium from these sources was $>40 \%$ of intake (about $1300 \mathrm{mg} /$ day). On average, a significant reduction in sodium intake (-561 mg, 95\% CI -891, -230) was achieved after 3 months. The main food sources contributing to the reduction included decreased consumption of processed foods $(\mathrm{p}<0.05)$ with a trend to reduced bread consumption $(\mathrm{p}<0.1)$. The reduction in sodium from processed foods amounted to $467 \mathrm{mg} /$ day $(>80 \%$ of the total).

Table 4 illustrates the changes in primary food sources of saturated fat over the duration of the PANDA trial. More than $20 \%$ of total saturated fat intake pre-intervention was from dairy products (cheese and milk). Added fat (butter, margarine and oils), fast foods and processed meats also contributed. Together, these foods accounted for $>40 \%$ of total saturated fat intake. Daily intake of saturated fat tended to decrease after 3 months $(-2.9$ g, 95\% CI $(-6.1,0.1))$ and of the food categories, milk (1-4\% fat) and processed meat consumption declined significantly at 3 months, together accounting for nearly $60 \%$ of the reduction.

Table 5 highlights the changes in primary food sources of added sugar in the PANDA intervention. At baseline, desserts (dairy-based or baked desserts) and yogurt were the main contributors to added sugar. Although, of those reporting yogurt, 18 participants out 
of 40 consumed fat-free yogurt with artificial sweeteners (data not shown). Chocolate, breakfast pastries and breakfast cereals were other main sources of added sugar. These foods accounted for $60 \%$ of total added sugar intake at baseline. There was a trend for added sugar intake to decrease $(p=0.097)$ at 3 months, which was accounted for mainly by significantly reduced consumption of pastries/baked desserts and chocolate.

\section{Discussion}

The main objectives of this study were to explore the primary food sources of sodium, saturated fat, and added sugar in people with diabetes in Canada and to determine if a menu plan-based intervention could reduce intakes of these nutrients. At baseline, intakes of sodium and saturated fat were above recommendations in this cohort, whereas added sugar met the recommendation of $\leq 10 \%$ of total energy. Overall, the intervention reduced total sodium and some sources of saturated fat consumption. Added sugar intake, already low, was not further reduced. Processed meats emerged as a leading source of both sodium and saturated fat. Other sources of sodium included other processed foods but also bread and grain-based mixed foods, while other sources of saturated fat included dairy products and added fats/oils along with fast food.

It is not surprising that only a quarter of diabetic patients had intakes below the UL for sodium (2300 mg/day) because sodium intake at baseline $(3200 \mathrm{mg} / \mathrm{d})$ was similar to the average of Canadian adults (3400 mg/d) (Health Canada, 2017). The primary source of dietary sodium in this cohort was bread, which is consistent with the general Canadian population (Garriguet, 2007), as well as diabetic patients from Spain (Guallar-Castillón et al., 2013). However, at $11 \%$ of total, the amount of sodium from bread was lower than 
that of Spanish diabetic populations (Guallar-Castillón et al., 2013) or general populations of the United Kingdom and United States (range 20-35\%) (Anderson et al., 2010). A lower proportion of sodium in bread in Canada may be explained by lower consumption of grains. About half of elderly Canadians consumed less than 5 daily servings of grain products (Canada Statistic, 2007), meanwhile; Americans consumed 10 servings of grains a day (USDA, 2005). Spaniards are reported to consume 37 $\mathrm{kg} /$ person/yr of bread (International Association of Plant Bakeries, 2015), which equates to $\sim 4$ slices per day (based on Canadian Nutrient File (Government of Canada, 2016), 1 slice of bread $=26 \mathrm{~g}$ ), suggesting that Spaniards eat almost twice as much bread as Canadians based on our cohort, which consumed an average of 2.3 servings/day. Although bread is not inherently rich in sodium, high consumption patterns make it a principle source of this nutrient as reported by Fischer (Fischer et al., 2009). As predicted, processed foods were other main sources of sodium. Intake attributed to processed meats $(10.8 \%$ of total) was similar to the Canadian population $(8.9 \%)$ (Fischer et al., 2009). We observed that sodium intake was significantly reduced after the PANDA intervention program by about $18 \%$, primarily because participants consumed less processed foods. Strategies from the PANDA menu plan that may have helped participants included recipes for homemade soups and limited use of condiments, along with lunch recipes using a variety of unprocessed protein sources, and use of herbs and spices to enhance flavor. Improved knowledge of food labeling (covered in the education modules) and increasing the number of homemade meals may have helped to reduce sodium intake in this population (Kitaoka et al., 2013). Although participants reduced sodium consumption, average intake still exceeded the UL of recommended intake. This 
outcome is similar to those of a 1-year dietary intervention focused on improving dietary quality among metabolic syndrome patients, which showed an overall reduction in sodium intake, but with more than half of participants' intake persistently exceeding the sodium UL (Wang et al., 2013). Lowering sodium intake has been associated with reductions in cardiovascular risk (Cook et al., 2014) and blood pressure (He et al., 2013); therefore it should be a priority in patients with type 2 diabetes. In the PANDA intervention program there was a significant reduction in systolic blood pressure (Asaad et al., 2016). However, we were not able to find an association between change in sodium intake and systolic blood pressure, probably because the study was not powered to detect this change. In 2007, Health Canada established a multi-stakeholder Sodium Working Group to develop strategies to reduce the sodium content of the diets of Canadians. The goal is to lower the average of sodium intake to $2300 \mathrm{mg}$ per day by 2016 (Health Canada, 2010). However, only $4.5 \%$ of Canadian food products had claims about sodium (Schermel et al., 2013). It has been estimated that the majority of sodium intake comes from processed foods (Fischer et al., 2009). Therefore, the food industry is seen to have a key role in reducing sodium intake from processed foods.

Several studies (Jarvandi et al., 2011; Muñoz-Pareja et al., 2012), including those from our group (Asaad and Chan, 2012; Asaad et al., 2015; Asaad et al., 2016) identify low adherence to recommendations for saturated fat consumption. Almost a quarter of dietary saturated fat in these diabetic patients came from dairy products (cheese and milk), similar to a Spanish diabetic population (Guallar-Castillón et al., 2013) and the general American population (Huth et al., 2013). After the intervention, we observed a significant reduction in saturated fat from milk due to a reduction of fat-containing milk (pre- 
intervention $0.8 \pm 0.9$ versus post-intervention $0.6 \pm 0.7$ servings/day, $\mathrm{p}<0.05$ ).

Consumption of non-fat milk products or alternatives did not increase despite the PANDA menu plan following Canada's Food Guide recommendation to consume 2-3 servings of low- or non-fat Milk and Alternatives daily (data not shown). The intervention also did not reduce the intake of saturated fat from cheese. One explanation is that consumers may not be willing to change to low-fat cheese because of perceived less desirable flavor and texture (Childs and Drake, 2009). Furthermore, the health risks and benefits of dairy are controversial. Two meta-analyses showed no association between dairy saturated fat and the risk of T2D (Chen et al. 2014; Gijsbers et al. 2016)., while other meta-analyses found an inverse association between cheese consumption and risk of T2D (Aune et al. 2013; Gao et al. 2013). Another main source of saturated fat was fats/oils, mostly butter and margarine. Saturated fat consumption from fats/oils was not significantly reduced in this trial; this may be due to the effect of fat on palatability and pleasure of the diet (Drewnowski, 1997). Fast foods also contributed as a main source of saturated fat. The Canadian Community Health Survey reported that one-quarter of Canadians had eaten something from fast food outlets daily (Statistics Canada, 2007), a similar proportion to the 15 out of 61 participants in our study. Following the menu plan did not reduce fast food intake, which might be due to its convenience and accessibility (Jekanowski et al., 2001); two-thirds of Edmonton's neighborhoods have at least one fast food outlet within 500 meters of their house (Smoyer-Tomic et al., 2008). As noted above, consumption of processed meats decreased over the intervention period. Recent meta-analysis data demonstrated that processed meats are associated with higher incidence of diabetes and coronary heart disease (Micha et al., 2010). Although 
participants tended to reduce saturated fat consumption from unhealthy food sources such as fast food and processed meat, healthier food sources of saturated fat such as yogurt and milk tended to increase. Thus nearly $90 \%$ of participants did not meet the recommended intake of saturated fat ( $<7 \%$ of energy intake) even after the intervention. This result indicates that diabetic patients have difficulty limiting saturated fat intake, which also as been documented in the general population of Canadian adults (Health Canada, 2012). Patients may lack knowledge about what kinds of foods contain high levels of fat (Asaad et al., 2015) or they may actually increase calories from fat after their diagnosis because of the emphasis on sugar/carbohydrate reduction (Yu et al., 2013).

Two-thirds of study participants met the recommended intake of added sugar $(\leq 10 \%$ of total energy) at baseline, which is consistent with the results of the 2004 Canadian Community Health Survey (CCHS) showing that diabetic respondents consumed less total sugar compared with non-diabetic respondents, possibly due to a focus on carbohydrate management for glycemic control (Langlois and Garriguet, 2012). The primary sources of total sugar were from fruits and vegetables, and dairy products (data not shown). Baked or dairy desserts represent the greatest contributors to dietary added sugar intake in this cohort. Although yogurt (a dairy dessert) is perceived to be a healthy food, about $75 \%$ of the total sugar is added sucrose or fructose in many varieties, which was determined by scrutinizing food labels (Durai Raj, 2017). Consumption of dairy desserts was not altered by the intervention, perhaps because yogurt was recommended as a healthier option than ice cream in the menu plan. Baked goods/desserts and chocolate were other sources of added sugar in diabetic patients and intakes of these were reduced significantly after the intervention. In our cohort of diabetic participants, sugar-sweetened 
beverages contributed little to added sugar intake (4.6\%), whereas in the general population of Canada (Langlois and Garriguet, 2012) and the United States (Huth et al., 2013), such beverages are major sources of added sugar (13\% and 33\%, respectively). There is conflicting advice about added sugar intake. The Canadian Diabetes Association (Dworatzek et al., 2013) and the World Health Organization (World Health Organization, 2014) suggest that added sugar intake should be $<10 \%$ of total calories; meanwhile, the American Diabetes Association (Franz et al., 2012) recommend $<25 \%$ of total calories from added sugar. Reductions in added sugar intake can be achieved through increases in healthy food choices that contain essential nutrients and fiber (e.g. fruit). Canadians with diabetes acquire more dietary sugars from fruit than the general population (Langlois and Garriguet, 2012) and after the PANDA intervention the Whole Fruits category of the Healthy Eating Index increased by $0.3 \pm 1.3$ serving $(p=0.059)$.

Although there were improvements in dietary intake, the majority of participants still had difficulty achieving recommended saturated fat and sodium intakes. Meeting these recommendations might be assisted by the development of strong public health policies for food industries to reduce saturated fat (Downs et al., 2013), sodium (Wang and Labarthe, 2015), and added sugar (Gornall, 2015) content in foods. In Canada, only 8.7\% of food products had claims about saturated fat, $4.5 \%$ claims about sodium, and $4 \%$ claims about sugar (Schermel et al., 2013). Currently, many people don't prioritize time for food preparation, thus contributing to changing food consumption patterns such as an increase in the consumption of fast foods and convenience or ready-to-eat foods. If the food industry increased the number of products with healthier profiles of saturated fat, 
sodium and added sugar, diabetic consumers would have greater access to appropriate foods (Jabs and Devine, 2006).

The main strength of this study is its novelty in identifying the primary food sources of sodium, saturated fat, and added sugar in Canadian diabetic patients. Additionally, dietary intake data were collected through validated methodology, multiple 24-hour recalls. We were able to evaluate how food sources of these nutrients changed following a menu plan-based intervention. The study had several limitations. Small sample size limits the ability to conduct multivariate analyses. The majority of participants lived in an urban area, were educated, Caucasian, and had high income/socio-economic status. Therefore, results may not be generalizable to those living in rural areas, with lower education levels, low income or different ethnicities. Additionally, dietary measurement error may have occurred due to inaccurate reporting by participants or limitations of the WebSpan database. For example, none of the participants reported added salt in their food intake, while WebSpan could not distinguish between homemade versus prepared food items. For example, nutrient analyses of soups are based on canned soups, which are likely to be higher in sodium content than homemade. The PANDA intervention encouraged preparation of foods "from scratch" by providing simple recipes; thus, the true extent of changes in nutrient intake may not have been captured. However, these limitations would be expected to dampen the true effects, thus conclusions regarding dietary changes are probably conservative.

In summary, the study highlights the main food sources of sodium, saturated fat, and added sugar in Canadian diabetic patients. Providing knowledge and skills regarding healthy food choices and menu planning through the PANDA intervention enabled 
healthy behavior changes resulting in significantly lower sodium intakes and a general reduction in processed foods such as meats and soups. However, continuing on a healthy diet may be challenging in terms of preventing diabetes complications. Although there were improvements in dietary intake, the majority of participants still had difficulty achieving recommended saturated fat and sodium intakes. Our findings can assist dietitians and other healthcare professionals in understanding dietary patterns of diabetic patients, which may enhance efforts to improve adherence to saturated fat, sodium and added sugar recommendations.

\section{Conflict of interest}

The authors declare no conflict of interest.

\section{Acknowledgments}

The authors thank the participants for their dedication to the study. We also thank Diana Soria-Contreras, who conducted the intervention education sessions.

\section{Financial Support}

Research funding was from Alberta Diabetes Institute, University of Alberta. GA received personal funding from the Ministry for Higher Education, Kingdom of Saudi Arabia.

\section{References}

Anderson, C.A., Appel, L.J., Okuda, N., Brown, I.J., Chan, Q., Zhao, L., Ueshima, H., Kesteloot, H., Miura, K., Curb. J.D., Yoshita, K., Elliott, P., Yamamoto, M.E., Stamler, J. 2010. Dietary sources of sodium in China, Japan, the United Kingdom, and the United 
States, women and men aged 40 to 59 years: the INTERMAP study. J. Am. Diet. Assoc. 110:736-745. doi: 10.1016/j.jada.2010.02.007. PMID: 20430135.

Asaad, G., and Chan, C.B. 2012. Relationship of diet quality to food security and nutrition knowledge in low-income, community-dwelling elders with type 2 diabetes mellitus: a pilot study. Can. J. Diabetes. 36:310-313. doi.org/10.1016/j.jcjd.2012.10.006. Asaad, G., Sadegian, M., Lau, R., Xu, Y., Soria-Contreras, D.C., Bell, R.C., Chan, C.B. 2015. The reliability and validity of the perceived dietary adherence questionnaire for people with type 2 diabetes. Nutrients. 7:5484-5496. doi:10.3390/nu7075231.

Asaad, G., Soria-Contreras, D.C., Bell, R.C., Chan, C.B. 2016. Effectiveness of a lifestyle intervention in patients with type 2 diabetes: The Physical Activity and Nutrition for Diabetes in Alberta (PANDA) trial. Health. Care. 4:73. doi: 10.3390/healthcare4040073. PMID: 27690122.

Aune, D., Norat, T., Romundstad, P., Vatten, L.J. 2013. Dairy products and the risk of type 2 diabetes: a systematic review and dose-response meta-analysis of cohort studies. Am. J. Clin. Nutr. 98(4): 1066-1083. doi: 10.3945/ajcn.113.059030. PMID: 23945722. Azadbakht, L., Fard, N.R., Karimi, M., Baghaei, M.H., Surkan, P.J., Rahimi, M., Esmaillzadeh, A., Willett, W.C. 2011. Effects of the Dietary Approaches to Stop Hypertension (DASH) eating plan on cardiovascular risks among type 2 diabetic patients: A randomized crossover clinical trial. Diabetes. Care. 34:55-57. doi: 10.2337/dc10-0676. PMID: 20843978.

Chen, M., Sun, Q., Giovannucci, E., Mozaffarian, D., Manson, J.E., Willett, W.C., Hu, F.B. 2014. Dairy consumption and risk of type 2 diabetes: 3 cohorts of US adults and an updated meta-analysis. BMC. Med. 12: 215. doi: 10.1186/s12916-014-0215-1. Childs, J., and Drake, M. 2009. Consumer perception of fat reduction in cheese. J Sens Stud. 24:902- 921. doi: 10.1111/j.1745-459X.2009.00243.x.

Cook, N., Appel, L., Whelton, P. 2014. Lower levels of sodium intake and reduced cardiovascular risk. Circulation. 9:981-989. doi:

10.1161/CIRCULATIONAHA.113.006032. PMID: 24415713.

Coyle, M., Francis, K., Chapman, Y. 2013. Self-management activities in diabetes care: a systematic review. Aust. Health. Rev. 37:513-522. doi: 10.1071/AH13060. PMID: 24018058. 
Downs, S., Thow, A., Leeder, S. 2013. The effectiveness of policies for reducing dietary trans fat: a systematic review of the evidence. Bull. World. Health. Organ. 91: 262-269H. PMID: 23599549.

Drewnowski, A. 1997. Taste preferences and food intake. Annu. Rev. Nutr. 17: 237-253. doi: 10.1146/annurev.nutr.17.1.237. PMID: 9240927.

Durai Raj GD, Hashemi Z, Soria Contreras DC, Babwik S, Maxwell D, Bell RC, Chan CB. 2017. Adherence to diabetes dietary guidelines assessed using a validated questionnaire predicts glucose control among individuals with type 2 diabetes. Can. J. Diabetes. 13: S1499. doi: 10.1016/j.jcjd.2017.04.006. PMID: 28648765.

Dworatzek, P.D., Arcudi, K., Gougeon, R., Husein, N., Sievenpiper, J.L., Williams, S.L. 2013. Nutrition therapy. Canadian Diabetes Association 2013 clinical practice guidelines for the prevention and management of diabetes in Canada. Can. J. Diabetes. 37: S54-S55. doi: 10.1016/j.jcjd.2013.01.009. PMID: 24070926.

Eilat-Adar, S., Xu, J., Zephier, E., O'Leary, V., Howard, B.V., Resnick, H.E. 2008. Adherence to dietary recommendations for saturated fat, fiber, and sodium is low in American Indians and other U.S. adults with diabetes. J. Nutr. 138:1699-1704. PMID: 18716172.

Ericson U, Hellstrand S, Brunkwall, L., Schulz, C., Sonestedt, E., Wallström, P., Gullberg, B., Wirfält, E., Orho-Melander, M. 2015. Food sources of fat may clarify the inconsistent role of dietary fat intake for incidence of type 2 diabetes. Am. J. Clin. Nutr. 101:1065-1080. doi: 10.3945/ajen.114.103010. PMID: 25832335.

Fischer, P., Vigneault, M., Huang R, Arvaniti, K., Roach, P. 2009. Sodium food sources in the Canadian diet. Appl. Physiol. Nutr. Metab. 5: 884-892. doi: 10.1139/H09-077. PMID: 19935850.

Franz, M.J. 2012. Macronutrients and nutrition therapy for diabetes. American Diabetes Association Guide to Nutrition Therapy for Diabetes. Edited by Franz, M.J., and Evert, A.B. Alexandria, VA, USA. p.p 19-40.

Garriguet, D. 2007. Sodium consumption at all ages. Health. Rep. 18: 47-52. PMID: 17578015.

Gao, D., Ning, N., Wang, C., Wang, Y., Li, Q., Meng, Z., Liu, Y., and Li, Q. 2013. Dairy products consumption and risk of type 2 diabetes: systematic review and dose-response 
meta-analysis. PloS one, 8(9): e73965. doi: 10.1371/journal.pone.0073965. PMID: 24086304.

Government of Canada. 2016. Canadian Nutrient File. Available from: https://foodnutrition.canada.ca/cnf-fce/index-eng.jsp [accessed 3 June 2017] Gijsbers, L., Ding, E.L., Malik, V.S., de Goede, J., Geleijnse, J.M., and SoedamahMuthu, S.S. 2016. Consumption of dairy foods and diabetes incidence: a dose-response meta-analysis of observational studies. Am. J. Clin. Nutr. doi: 10.3945/ajen.115.123216. PMID: 26912494.

Goede, J. D., Geleijnse, J.M., Ding, E.L., Soedamah-Muthu, S.S. 2015. Effect of cheese consumption on blood lipids: a systematic review and meta-analysis of randomized controlled trials. Nutrition. Reviews. 73: 259-275. doi: 10.1093/nutrit/nuu060. PMID: 26011901.

Gornall, J. 2015. Sugar: spinning a web of influence. Br. Med. J. 15: h231. doi: 10.1136/bmj.h231. PMID: 25673325.

Guallar-Castillón, P., Muñoz-Pareja, M., Aguilera, M.T., León-Muñoz, L.M., RodríguezArtalejo, F. 2013. Food sources of sodium, saturated fat and added sugar in the Spanish hypertensive and diabetic population. Atherosclerosis. 229: 198-205. doi:

10.1016/j.atherosclerosis.2013.04.001. PMID: 23642731.

He, F., Li, J., Macgregor, G. 2013. Effect of longer term modest salt reduction on blood pressure: Cochrane systematic review and meta-analysis of randomised trials. Br. Med. J. 7903:1-12. doi: 10.1136/bmj.f1325. PMID: 23558162.

Health Canada. 2006. Canadian community health survey, Cycle 2.2 nutrition. Available from: http://www.hc-sc.gc.ca/fn-an/surveill/nutrition/commun/cchs_guide_escc-eng.php [accessed 6 March 2017]

Health Canada. 2010. Sodium Reduction Strategy for Canada. Available from: http://www.hc-sc.gc.ca/fn-an/nutrition/sodium/related-info-connexe/strateg/reduct-strateng.php [accessed 10 April 2017]

Health Canada. 2012. Do Canadian adults meet their nutrition requirements through food intake alone? Available from: http:/www.hc-sc.gc.ca/fnan/surveill/nutrition/commun/art-nutr-adult-eng.php\#a322 [accessed 6 March 2017] 
Health Canada. 2017. Sodium in Canada. Available from: http://www.hc-sc.gc.ca/fnan/nutrition/sodium/index-eng.php [accessed 6 march 2017].

Huth, P.J., Fulgoni, V.L., Keast, D.R., Park, K., Auestad, N. 2013. Major food sources of calories, added sugars, and saturated fat and their contribution to essential nutrient intakes in the U.S. diet: data from the National Health and Nutrition Examination Survey (20032006). J. Nutr. 12: 116-126. doi: 10.1186/1475-2891-12-116. PMID: 23927718.

International Association of Plant Bakeries. 2015. Bread Market Report 2013. Available from http://www.aibi.eu/wp-content/uploads/draft-AIBI-Bread-Market-report-2013.pdf [accessed 3 June 2017]

Jabs, J., and Devine, C. 2006. Research review: Time scarcity and food choices: An overview. Appetite. 47:196-204. doi: 10.1016/j.appet.2006.02.014. PMID: 1669811. Jarvandi, S., Gougeon, R., Bader, A., Dasgupta, K. 2011. Differences in food intake among obese and nonobese women and men with type 2 diabetes. J. Am. Coll. Nutr. 30: 225-232. dio: 10.1080/07315724.2011.10719964. PMID: 21917702.

Jekanowski, M.D., Binkley, J.K., Eales, J. 2001. Convenience, accessibility, and the demand for fast food. J. Agr. Resour. Econ. 1: 58-74.

Jin, J., Sklar, G.E., Min Sen Oh.V., Li, C.S. 2008. Factors affecting therapeutic compliance: A review from the patient's perspective. Ther. Clin. Risk .Manag. 4: 269286.

Kitaoka, K., Nagaoka, J., Matsuoka, T., Shigemura, C., Harada, K., Aoi, W., Wada, S., Asano, H., Sakane, N., Higashi, A. 2013. Dietary intervention with cooking instructions and self-monitoring of the diet in free-living hypertensive men. Clin. Exp. Hypertens. 35: 120-127. doi: 10.3109/10641963.2012.702830. PMID: 22799766.

Langlois, K., and Garriguet, D. 2012. Sugar consumption among Canadians of all ages. Health. Rep. 3: 1-5. PMID: 22106786.

Ley, S.H., Pan, A., Li, Y., Manson, J.E., Willett, W.C., Sun, Q., Hu, F.B. 2016. Changes in overall diet quality and subsequent type 2 diabetes risk: Three U.S. prospective cohorts. Diabetes. Care. 39: 2011-2018.

McEvoy, C.T., Cardwell, C.R., Woodside, J.V., Young, I.S., Hunter, S.J., McKinley, M.C. 2014. A posteriori dietary patterns are related to risk of type 2 diabetes: findings 
from a systematic review and meta-analysis. J. Acad. Nutr. Diet. 114: 1759-1775.e4. doi: 10.1016/j.jand.2014.05.001. PMID: 25001435.

Micha, R., Wallace, S., Mozaffarian, D. 2010. Red and processed meat consumption and risk of incident coronary heart disease, stroke, and diabetes mellitus: a systematic review and meta-analysis. Circulation. 21: 2271-2283. doi:

10.1161/CIRCULATIONAHA.109.924977.

Muñoz-Pareja, M., Leo'n-Muñoz, L.M., Guallar-Castillo'n, M., Graciani, A., Lo'pezGarc1'a, E., Banegas, J.R., Rodri'guez-Artalejo, F. 2012. The diet of diabetic patients in Spain in 2008-2010: accordance with the main dietary recommendations a cross-sectional study. PloS one, 7:1-9. doi: 10.1371/journal.pone.0039454.

National Academy of Sciences. 1988. Animal product options in the marketplace. National Academy Press (US), Washington D.C. Available from: http://www.ncbi.nlm.nih.gov/books/NBK218184/[accessed 10 April 2017] Rippe, J.M., and Angelopoulos, T. J. 2016. Relationship between Added Sugars Consumption and Chronic Disease Risk Factors: Current Understanding. Nutrients, 8(11), 697. doi: 10.3390/nu8110697. PMID: 27827899.

Rivellese, A.A., Boemi, M., Cavalot, F., Costagliola, L., De Feo, P., Miccoli, R., Patti, L., Trovati, M., Vaccaro, O., Zavaroni, I., Mind.it Study Group. 2008. Dietary habits in type II diabetes mellitus: how is adherence to dietary recommendations?. Eur. J. Clin. Nutr. 62: 660-664. doi: 10.1038/sj.ejcn.1602755. PMID: 17426738.

Schermel, A., Emrich, T.E., Arcand, J., Wong, C.L., L'Abbé, M.R. 2013. Nutrition marketing on processed food packages in Canada: 2010 Food Label Information Program. Appl. Physiol. Nutr. Metab. 38: 666-672. doi: 10.1139/apnm-2012-0386. PMID: 23724885.

Shi, Y., de Groh, M., Morrison, H., Robinson, C., Vardy, L. 2011. Dietary sodium intake among Canadian adults with and without hypertension. Chronic. Dis. Can. 31: 79-87. PMID: 21466758.

Smoyer-Tomic, K.E., Spence, J.C., Raine, K.D., Amrhein, C., Cameron, N., Yasenovskiy, V., Cutumisu, N., Hemphill, E., Healy, J. 2008. The association between neighborhood socioeconomic status and exposure to supermarkets and fast food 
outlets. Health. Place. 14:740-754. doi: 10.1016/j.healthplace.2007.12.001. PMID: 18234537.

Soedamah-Muthu, S.S., Ding, E.L., Al-Delaimy, W.K., Hu, F.B., Engberink, M.F., Willett, W.C., Geleijnse, J.M. 2011. Milk and dairy consumption and incidence of cardiovascular diseases and all-cause mortality: dose-response meta-analysis of prospective cohort studies. Am. J .Clin. Nutr. 93: 158-171. doi: 10.3945/ajen.2010.29866. PMID: 21068345.

Soria-Contreras, D.C., Bell, R.C., McCargar, L.J., Chan, B.C. 2014. Feasibility and efficacy of menu planning combined with individual counselling to improve health outcomes and dietary adherence in people with type 2 diabetes: a pilot study. Can. J. Diabetes. 38: 320-325. doi: 10.1016/j.jcjd.2014.03.009. PMID: 25175312.

Statistics Canada. 2007. Canadians' eating habits. Available from:

http://www.statcan.gc.ca/pub/82-003-x/2006004/article/habit/4148989-

eng.htm. [accessed 6 March 2017].

Storey, K., and McCargar L.J. 2012. Reliability and validity of Web-SPAN, a web-based method for assessing weight status, diet and physical activity in youth. J. Hum. Nutr.

Diet. 25: 59-68. doi: 10.1111/j.1365-277X.2011.01181.x. PMID: 21615806.

Stratton, I.M., Adler, A.I., Neil, H.A., Matthews, D.R., Manley, S.E., Cull, C.A., Hadden, D., Turner, R.C., Holman, R.R. 2000. Association of glycaemia with macrovascular and microvascular complications of type 2 diabetes (UKPDS 35): prospective observational study. Br. Med. J. 321: 405-412. doi: https://doi.org/10.1136/bmj.321.7258.405. PMID: 10938048.

Thanopoulou, A., Karamanos, B., Angelico, F., Assaad-Khalil, S., Barbato, A., Del Ben, M., Djordjevic, P., Dimitrijevic-Sreckovic, V., Gallotti, C., Katsilambros, N., Migdalis, I., Mrabet, M., Petkova, M., Roussi, D., Tenconi, M.T. 2004. Nutritional habits of subjects with Type 2 diabetes mellitus in the Mediterranean Basin: comparison with the non-diabetic population and the dietary recommendations. Multi-Centre Study of the Mediterranean Group for the Study of Diabetes (MGSD). Diabetologia, 47: 367-376. doi: 10.1007/s00125-003-1316-0. PMID: 14730377. 
USDA. 2005. Americans' Whole-Grain Consumption Below Guidelines. Available from: https://www.ers.usda.gov/amber-waves/2005/april/americans-whole-grain-consumptionbelow-guidelines/. [accessed 22 June 2017].

Wang, G., and Labarthe, D. 2011. The cost-effectiveness of interventions designed to reduce sodium intake. J. Hypertens. 29: 1693-1699. doi:

10.1097/HJH.0b013e328349ba18. PMID: 21785366.

Wang, J., Olendzki, B.C., Wedick, N.M., Persuitte, G.M., Culver, A.L., Li, W., Merriam, P.A., Carmody, J., Fang, H., Zhang, Z., Olendzki, G.F., Zheng, L., Ma, Y. 2013.

Challenges in sodium intake reduction and meal consumption patterns among participants with metabolic syndrome in a dietary trial. J. Nutr. 12: 163-171. doi: 10.1186/1475-289112-163. PMID: 24345027.

Willet, W. 1998. Nutritional Epidemiology, 2nd edition; Oxford University Press: New York, NY, USA.

Wolfson, J., and Bleich, S. 2015. Is cooking at home associated with better diet quality or weight-loss intention? Public. Health. Nutr. 18:1397-1406. doi:

10.1017/S1368980014001943. PMID: 25399031.

World Health Organization. 2014. The science behind the sweetness in our diets.

Available from: http://www.who.int/bulletin/volumes/92/11/14-031114.pdf [accessed 6 March 2017].

Yu, M., Ailawadi, K.L., Grewal, D. 2013. Soda versus cereal and sugar versus fat: drivers of healthful food intake and the impact of diabetes diagnosis. J. Mark. 77:101-120. doi: 10.1509/jm.11.0443. 
Table 1. Food classification for food sources

\begin{tabular}{|c|c|c|}
\hline Code & Food group & Components \\
\hline 1 & Bread & Bread/ bagels/ buns \\
\hline 2 & Breakfast cereals & Wheat cereal/corn cereal/rice cereal/oatmeal \\
\hline 3 & Breakfast pastries & Muffins/cinnamon buns/waffles/pancakes/granola bars \\
\hline 4 & Pasta/rice/grains & Pasta/noodles/rice/quinoa/couscous \\
\hline 5 & Grain snacks & Crackers/pretzels \\
\hline 6 & Milk & $1-4 \%$ fat milk \\
\hline 7 & $\begin{array}{l}\text { Non-fat milk \& } \\
\text { alternatives }\end{array}$ & Skim milk/rice milk/soy milk \\
\hline 8 & Chocolate milk & $2 \%$ chocolate milk $/ 1 \%$ chocolate milk \\
\hline 9 & Dairy desserts/ yogurt & Yogurt/ice cream/smoothies/pudding/sour cream \\
\hline 10 & Cheese & Fresh cheese/cured cheese/cheese spread \\
\hline 11 & Processed meat & Sausage/luncheon meat/ham \\
\hline 12 & Fish & Fresh fish/canned fish \\
\hline 13 & Seafood & Crustaceans/molluscs \\
\hline 14 & White meat & Poultry \\
\hline 15 & Eggs & \\
\hline 16 & Red meat & Veal/beef/pork/lamb \\
\hline 17 & Meat alternatives & Nuts/dried beans/lentils \\
\hline 18 & Fruits \& vegetables & Fresh/dried \\
\hline 19 & Vegetables juices & Vegetables /tomato juice \\
\hline 20 & Sweetened fruits & Sweetened fruit juices, Canned fruits \\
\hline 21 & Fruit juices & Unsweetened fruit juices \\
\hline 22 & Condiments & Ketchup/mustard/mayonnaise/pickles/relish/gravy/salsa \\
\hline 23 & Asian sauces & Soy sauce/Teriyaki sauce \\
\hline 24 & Soft drinks & Sugar-sweetened pops/energy drinks \\
\hline 25 & Liquor & Beer/wine \\
\hline 26 & Sugar/honey/syrup & White sugar/brown sugar/maple syrup/pancake syrup \\
\hline 27 & Jam/jelly & Fruit jam/fruit jelly \\
\hline 28 & Baked goods/desserts & Cookies/cake/ brownies/pies \\
\hline 29 & Chocolate & Chocolate bars/chocolate spread/fudge \\
\hline 30 & Candy & Hard candy/sugary chewing gum/ licorice candy \\
\hline 31 & Dressing/dip & Salad dressing/ hummus \\
\hline 32 & Fats and oils & Oils/butter/margarine \\
\hline 33 & Chips & Cheesies/potato chips/tortilla chips \\
\hline 34 & Fast food & Beef and chicken hamburgers/hotdogs/French fries/onion rings/chicken fingers \\
\hline 35 & Pizza & \\
\hline 36 & Sandwiches \& wraps & \\
\hline 37 & Soups & \\
\hline 38 & Mixed food meat based & Meat pies/stir fries/stews \\
\hline 39 & Mixed food grain based & Sushi/fried rice/perogies/pasta dishes/nachos with toppings \\
\hline 40 & $\begin{array}{l}\text { Mixed food vegetable } \\
\text { based }\end{array}$ & Vegetarian chilis and stir fries \\
\hline
\end{tabular}


Table 2. Average of sodium, saturated fat and added sugar intakes in the PANDA intervention study $\mathrm{N}=61$

\begin{tabular}{|l|l|l|l|l|}
\hline & Pre-intervention & $\begin{array}{l}\text { Post- } \\
\text { intervention }\end{array}$ & p-value* & Recommendation \\
\hline Sodium (mg/ day) & $3281 \pm 1348$ & $2720 \pm 973$ & 0.001 & $<2300 \mathrm{mg} /$ day $^{\mathrm{a}}$ \\
\hline $\begin{array}{l}\text { Sodium density (sodium } \\
\text { mg /1000 kcal) }\end{array}$ & $1.6 \pm 0.5$ & $1.4 \pm 0.3$ & 0.006 & \\
\hline Saturated fat (g/day) & $26.8 \pm 11.1$ & $23.9 \pm 10.8$ & 0.058 & \\
\hline Saturated fat (\% TE) & $11.4 \pm 2.7$ & $10.8 \pm 3.1$ & 0.154 & $<7 \% \mathrm{TE}^{\mathrm{b}}$ \\
\hline Added sugar (g/day) & $47.1 \pm 38$ & $40.1 \pm 24.2$ & 0.097 & \\
\hline Added sugar (\%TE) & $8.7 \pm 4.8$ & $8.3 \pm 4.0$ & 0.492 & $<10 \% \mathrm{TE}^{\mathrm{b}}$ \\
\hline
\end{tabular}

a- Health Canada (2017) upper daily limit

b - Canadian Diabetes Association clinical practice guidelines 2013

*Paired t-test comparisons for each assessment, $\mathrm{p}<0.05$ was considered significant 
Table 3. Effect of the PANDA intervention on intake of sodium from the top five food sources

\begin{tabular}{|l|l|l|l|l|}
\hline Food sources of sodium & $\begin{array}{l}\text { Contribution at } \\
\text { baseline } \% \text { of } \\
\text { total })\end{array}$ & $\begin{array}{l}\text { Pre-intervention } \\
\text { (mg/day) }\end{array}$ & $\begin{array}{l}\text { Post-intervention } \\
\text { (mg/day) }\end{array}$ & p-value* \\
\hline Bread & $11.4 \%$ & $450 \pm 352$ & $318 \pm 195$ & 0.053 \\
\hline Processed meats & $10.8 \%$ & $370 \pm 347$ & $193 \pm 240$ & 0.002 \\
\hline Soup & $10.3 \%$ & $385 \pm 589$ & $210 \pm 303$ & 0.014 \\
\hline Condiments & $6.4 \%$ & $193 \pm 234$ & $94 \pm 180$ & 0.007 \\
\hline Mixed food grain based & $5.4 \%$ & $178 \pm 320$ & $212 \pm 293$ & 0.533 \\
\hline
\end{tabular}

*Paired t-test comparisons for each assessment, $\mathrm{p}<0.05$ was considered significant 
Table 4. Effect of the PANDA intervention on intake of saturated fat from the top five food sources

\begin{tabular}{|l|l|l|l|l|}
\hline $\begin{array}{l}\text { Food sources of saturated } \\
\text { fat }\end{array}$ & $\begin{array}{l}\text { Contribution at } \\
\text { baseline }(\% \text { of } \\
\text { total })\end{array}$ & $\begin{array}{l}\text { Pre-intervention } \\
\text { (g/day) }\end{array}$ & $\begin{array}{l}\text { Post-intervention } \\
\text { (g/day) }\end{array}$ & p-value* \\
\hline Cheese & $12 \%$ & $3.5 \pm 3.8$ & $3.3 \pm 4.1$ & 0.865 \\
\hline Milk (1-4\% fat) & $10 \%$ & $2.7 \pm 4.2$ & $1.7 \pm 2.1$ & 0.052 \\
\hline Fats/oils & $9.1 \%$ & $2.7 \pm 3.4$ & $2.5 \pm 3.6$ & 0.710 \\
\hline Fast food & $7.4 \%$ & $1.9 \pm 2.7$ & $1.4 \pm 3.1$ & 0.263 \\
\hline Processed meats & $5.7 \%$ & $1.7 \pm 1.9$ & $1.0 \pm 1.7$ & 0.038 \\
\hline
\end{tabular}

*Paired t-test comparisons for each assessment, $\mathrm{p}<0.05$ was considered significant 
Table 5. Effect of the PANDA intervention on intake of added sugar from the top five food sources

\begin{tabular}{|l|l|l|l|l|}
\hline Food source & $\begin{array}{l}\text { Contribution at } \\
\text { baseline }(\% \text { of } \\
\text { total) }\end{array}$ & $\begin{array}{l}\text { Pre-intervention } \\
(\mathrm{g} / \text { day })\end{array}$ & $\begin{array}{l}\text { Post-intervention } \\
(\mathrm{g} / \text { day })\end{array}$ & $\mathrm{p}$-value* \\
\hline Dairy dessert/yogurt & $17.3 \%$ & $7.7 \pm 9.4$ & $9.3 \pm 11.7$ & 0.296 \\
\hline Baked goods/desserts & $13.5 \%$ & $6.5 \pm 9.7$ & $3.8 \pm 6.1$ & 0.029 \\
\hline Chocolate & $10.9 \%$ & $2.4 \pm 6.6$ & $0.4 \pm 1.6$ & 0.026 \\
\hline Breakfast pastries & $8.9 \%$ & $3.9 \pm 5.9$ & $3.3 \pm 6.3$ & 0.568 \\
\hline Breakfast cereals & $8.8 \%$ & $3.2 \pm 5.5$ & $3.4 \pm 5.2$ & 0.796 \\
\hline
\end{tabular}

*Paired t-test comparisons for each assessment, $\mathrm{p}<0.05$ was considered significant 